\title{
Glucocorticoid Regulation of Stress-Induced Mineralocorticoid Receptor Gene Transcription in Vivo
}

\author{
JAMES P. HERMAN ${ }^{a}$ AND STANLEY J. WATSON ${ }^{b}$ \\ ${ }^{a}$ Department of Anatomy and Neurobiology \\ University of Kentucky Medical Center \\ 800 Rose Street \\ Lexington, Kentucky 40536-0084 \\ and \\ ${ }^{b}$ Mental Health Research Institute \\ University of Michigan \\ 205 Zina Pitcher Place \\ Ann Arbor, Michigan 48109-0720
}

The neuronal mineralocorticoid receptor (MR) binds endogenous corticosterone (B) with high affinity. ${ }^{1}$ The ligand-activated MR is transported to the cell nucleus, where it is capable of affecting transcription of a wide variety of genes. ${ }^{2}$ Within the central nervous system, the MR is of highest abundance within the hippocampus, thus leading to its hypothetical association with hippocampally mediated negative feedback control of the hypothalamo-pituitary-adrenocortical (HPA) stress axis. ${ }^{3,4}$

Understanding regulation of the neuronal MR is critical to elucidating its true function within the nervous system. In the present experiments, we employed a semiquantitative in situ hybridization strategy to assess in vivo stress regulation of neuronal MR mRNA and gene expression. For characterization of the MR intron probe, brains of normal Sprague-Dawley rats were sectioned at $20 \mu \mathrm{m}$ and sections through the hippocampus processed for in situ hybridization. Adjacent series were hybridized with probes complementary to 1 ) the $3^{\prime}$ coding and proximal $3^{\prime}$ untranslated region of the MR mRNA (exon probe) and 2 ) the $5^{\prime}$ portion of a coding region intron lying between exons 2 and 3 (intron probe). The latter probe was designed to recognize unspliced or partially-spliced gene transcripts present in the cell nucleus as heteronuclear (hn) RNA. An additional series was preincubated with RNAse A to verify probe specificity. Sections were dipped in NTB2 liquid emulsion and counterstained with cresyl violet. For analysis of stress and glucocorticoid effects on MR gene and mRNA regulation, rats received either adrenalectomy (ADX) or sham operation (SHAM), and were subsequently implanted with pellets containing either cholesterol (ADX-C and SHAM-C rats) or 30\% B/70\% cholesterol (ADX-B rats). Acute restraint stress (plastic restraint cages, $60 \mathrm{~m}$ ) was imposed 5 days following pellet implantation, and groups of animals were killed 60 and $120 \mathrm{~m}$ post-stress, with unstressed rats serving as controls. Blood samples from all rats were assayed for $B$.

Localization of signal generated by MR exon and intron probes are shown in FIGURE 1. MR exon signal was located diffusely throughout the cell (FIG. 1A,B) and showed a characteristic distribution across hippocampal subfields and dentate gyrus (FIG. 1C). ${ }^{5-7}$ Cytoplasmic localization was confirmed on Nissl-counterstained sections (data not shown). In contrast, the MR intron probe showed a punctate distribution consistent with nuclear localization (FIG. 1D,E). MR intron signal (FIG. 1F) could be localized to all hippocampal subfields, and was significantly less intense than exon signal at equal exposure times. The localization data indicate 1) compart- 

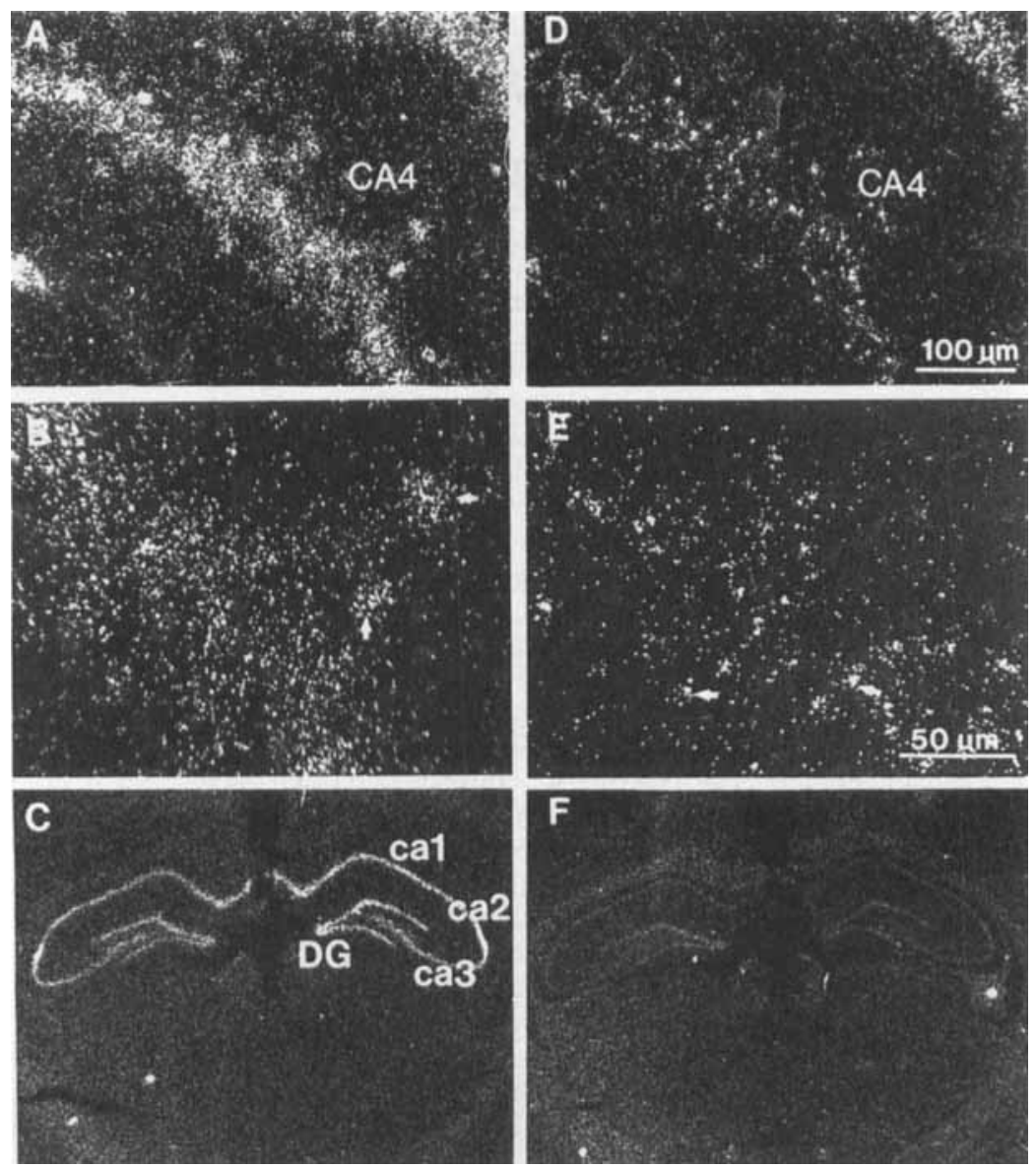

FIGURE 1. Localization of MR mRNA (A-C) and hnRNA (D-F) in the hippocampal formation of the rat. MR mRNA, as detected by a probe complementary to sequences encoding the protein coding region and $3^{\prime}$-untranslated domain, can be seen as a scattering of grains over hippocampal neurons (A). Individual neurons show diffuse labeling over hippocampal cells (B). MR mRNA can be localized to all hippocampal subfields and the dentate gyrus (DG) (C). MR hnRNA, as detected using a coding region intron probe, can be appreciated as punctate aggregations of grains consistent with nuclear localization (D,E). Like MR mRNA, MR hnRNA is localized to all hippocampal subfields, albeit at significantly lower abundance (F vs C). Note that (C) and (F) are adjacent sections through the hippocampus of a normal rat, and have been exposed for the same period of time ( 9 days on Kodak XAR5 film).

mentalization of intron signal within the nucleus, consistent with detection of unspliced or partially spliced transcripts and 2) low resting levels of the intronic sequence, consistent with a high ratio of mature message to primary or partially processed gene transcripts.

The B data verify the efficacy of the stress/replacement regimens. SHAM-C rats 
showed a predictable stress response (time $0,40.9 \pm 16.7 \mathrm{ng} / \mathrm{ml}$; time $60 \mathrm{~m}, 284.7$ $\pm 67.2 \mathrm{ng} / \mathrm{ml}$; time $120 \mathrm{~min} 21.3 \pm 11.4 \mathrm{ng} / \mathrm{ml}$ ). In contrast, ADX-CORT rats showed slightly high basal B levels which did not change in response to stress (time $0,49.6$ $\pm 18.5 \mathrm{ng} / \mathrm{ml} ;$ time $60 \mathrm{~m}, 47.7 \pm 7.5 \mathrm{ng} / \mathrm{ml} ;$ time $120 \mathrm{~min} 59.2 \pm 27.6 \mathrm{ng} / \mathrm{ml}$ ).

In response to acute stress, MR hnRNA decreased to some $50 \%$ of unstressed baseline in SHAM-C rats in subfield CA1, indicative of a rapid inhibition of MR gene transcription following stress in these hippocampal regions (FIG. 2). ADX-B rats, on the other hand, showed no significant change in MR intron hybridization

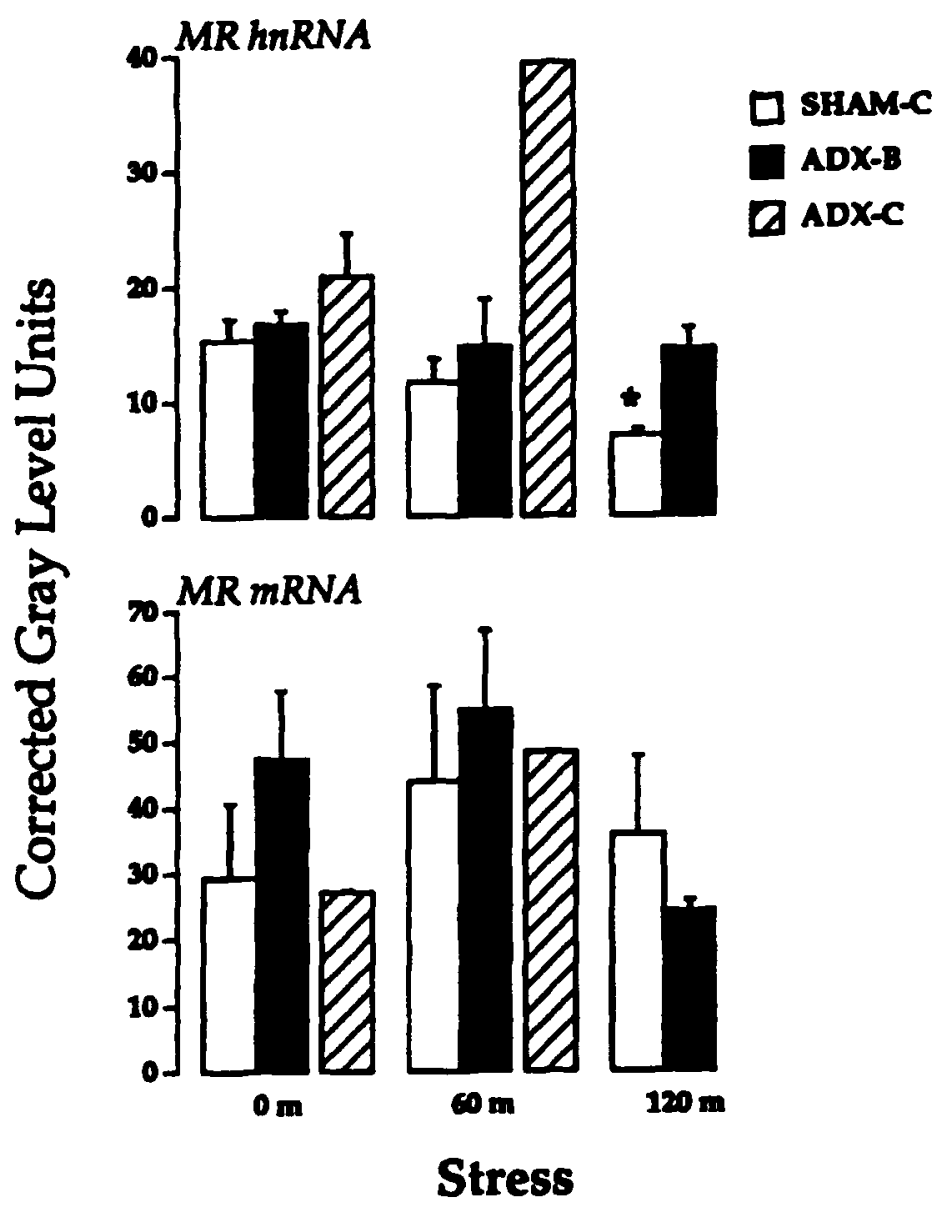

FIGURE 2. Regulation of MR hnRNA and MR mRNA in subfield CA1 of the hippocampus. Two-way ANOVA revealed a significant effect of group on MR hnRNA expression $(p<0.05)$ and a significant interaction effect $(p<0.05)$. Subsequent comparison among means revealed a significant difference between SHAM-C time 0 and SHAM-C time 120, and ADX-B time 120 and SHAM-C time 120. Results indicate that the observed stress-induced decrease in MR hnRNA expression is likely to stem from adrenal glucocorticoid release. 
following stress. These results indicate that in the absence of the glucocorticoid flux normally seen following stress, the MR gene does not downregulate. The adrenal stress response is therefore required for normal MR downregulation to occur.

Hybridization with the MR exon probe revealed no significant downregulation by stress in SHAM-C rats or ADX-B rats over the 2-hour post-stress period. These data raise the possibility that steady-state levels of MR mRNA are stable over this time period, resulting in no decrement in message despite decreased transcription, or alternatively that the signal generated by the stress episode acts to stabilize cytoplasmic mRNA. The latter possibility is highlighted by the trend toward a decrease in MR mRNA seen in ADX-B rats at the 2-hour post-stress time point (FIg. 2).

The rapid downregulation of hippocampal MR gene transcription following stress indicates a keen sensitivity of the MR to glucocorticoid fluxes. This observation implies a sensitivity of MR-mediated transcriptional events to stressful information, and further implies a role for the MR in hippocampal functions, including general information processing and possibly HPA regulation. Further analysis is required to determine how transcriptional changes affect receptor protein function and/or selection of MR alternative splicing products. ${ }^{8}$

\section{REFERENCES}

1. Reul, J. M. \& E. R. DE KLoet. 1985. Endocrinology 117: 2505-2511.

2. Herman, J. P. 1993. Cell. Mol. Neurobiol. 13: 349-372.

3. DE Kloet, E. R. 1992. Ann. N.Y. Acad. Sci. 663: 357-371.

4. Jacobson, L. \& R. M. Sapolsky. 1990. Endocr. Rev. 12: 118-134.

5. Herman, J. P., P. D. Patel, H. Akil \& S. J. Watson. 1989. Mol. Endocrinol. 3: 1886-1894.

6. Arriza, J. L., R. B. Simerly, L. W. Swanson \& R. M. Evans. 1988. Neuron 1: $887-900$.

7. Vaneekelen, J. A. M., W. Jiang, E. R. de Kloet \& M. C. Bohn. 1988. J. Neurosci. Res. 21: 88-94.

8. Kwak, S. P., P. D. Patel, R. C. Thompson, H. Akil \& S. J. Watson. 1993. Endocrinology 133: 2344-2350. 